\title{
LAS CONCLUSIONES DE LOS ARTÍCULOS DE INVESTIGACIÓN EN HISTORIA*
}

\author{
Miguel Fuentes Cortés**
}

\begin{abstract}
Resumen
A pesar de los numerosos estudios de Movidas Retóricas en Artículos de Investigación, existen escasos estudios sobre la sección conclusiones en la disciplina de la historia. En este contexto, el objetivo de nuestra investigación es analizar las conclusiones de los Artículos de Investigación en historia en un corpus de revistas de categoría ISI en Suramérica. Los resultados permiten comprobar que la sección conclusiones no es una sección que se presenta en un $100 \%$ en historia, y, además, que la Movida Retórica más típica de esta sección es interpretar resultados. Nuestro objeto de estudio sirve de soporte para la comprensión de este género en historia, además de ser una herramienta para la enseñanza de la escritura científica.
\end{abstract}

Palabras clave: movidas retóricas, discurso científico de la historia, artículos de investigación, conclusiones.

\section{THE CONCLUSION SECTION IN THE HISTORY RESEARCH}

\section{ARTICLES}

\begin{abstract}
Despite the numerous studies of Rhetorical Moves in Research Articles, there are few studies on the conclusions section in the discipline of history. In this context, the aim of our research is to analyze the conclusions section of Research Articles in history in a corpus of ISI category journals in South America. The results allow us to prove that the conclusions section does not appear in a $100 \%$ in history, and also that the most typical rhetoric move in this section is to interpret results. Our object of study supports the understanding of this genre in history, besides being a tool for teaching scientific writing.
\end{abstract}

Keywords: rethorical moves, scientific discourse of history, research articles, conclusions.

Recibido: 19-12-2012 Aceptado: 03-06-2013

* Esta investigación se realizó en el marco del proyecto FONDECYT 11080097: El artículo de investigación a través de las disciplinas: El caso del indexador Scielo Chile.

** Chileno, Profesor de Historia y Geografía y Licenciado en Educación de la Universidad de La Serena, Magíster en Estudios Latinoamericanos, mención Lingüística de la ULS. Docente en la ULS, Investigador asociado a la Dirección de Investigación de la ULS y a Prodicyt, Centro de Investigación.mfuentes@userena.cl 


\section{Introducción}

La conclusión es la sección final de un Artículo de Investigación (en adelante AI). Es decir, donde uno esperaría el cierre del AI. Las conclusiones han sido estudiadas desde la narrativa (Propp, 1974), desde la teoría de la valoración (Appraisal) (Ferrari, 2006), y desde el análisis de género (Gnutzmann \& Oldenburg, 1991; Ciapuscio \& Otañi, 2002). Algunos de estos estudios han identificado que las conclusiones no se presentan en un 100\% en los AI (Gnutzmann \& Oldenburg, 1991; Ciapuscio \& Otañi, 2002; Fuentes, 2012). Otros autores han propuesto manuales de escritura científica, donde dan indicaciones de cómo escribir esta sección (Henríquez \& Zepeda, 2004; Valderrama, 2005, 2009; Cázares y otros, 1999; Sánchez, 2011). Por otro lado, y específicamente, desde el análisis de género, el discurso histórico ha sido escasamente estudiado, exceptuando los trabajos de Holmes (1997) y Fuentes (2012). De esta manera, el objetivo de nuestra investigación es analizar las conclusiones de los AI en historia en un corpus de revistas suramericanas indexadas en ISI. Después de esta introducción, el texto sigue con un capítulo teórico. A continuación, se exponen los procedimientos, seguidos de los resultados y la discusión. En la última parte, se muestran las principales conclusiones de la investigación.

\section{Marco teórico}

En la presente sección describimos los principales aspectos teóricos de nuestra investigación. Para esto, profundizamos en los temas asociados al discurso histórico, los estudios de género en el discurso histórico, un análisis multifuncional sobre las conclusiones, y finalmente la elaboración de un modelo de Movidas Retóricas (en adelante MR) para las conclusiones en historia.

\subsection{Características del discurso histórico}

La palabra historia presenta al menos tres significados: a) Narración o relato de un hecho pasado, b) Hecho pasado, y c) ciencia o disciplina que estudia el pasado (Koselleck, 2010). Esta polisemia influye directamente en la forma de cómo se escribe en un género científico la historia. Epistemológicamente, en la actualidad, e independiente del género, la mayoría de los estudios coinciden en el carácter esencialmente narrativo del discurso histórico (Ortega y Gasset, 1983; Veyne, 1984; Danto, 1989;

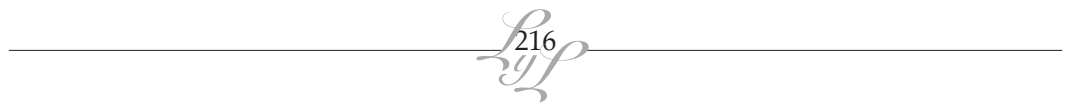


Lozano, 1994; Chartier, 1994; Ordóñez, 2008; White, 2003, 2010; Fuentes, 2012). Otros estudios reivindican el carácter argumentativo, o la tendencia a la persuasión que tiene el contenido del discurso histórico (Mudrovcic, 2005). Estos dos aspectos son esenciales en el discurso histórico, en tanto, la forma de transmitir el conocimiento no puede evitar una forma narrativa, ni tampoco, evitar la pretensión de convencer a la audiencia sobre las causas y consecuencias de un determinado hecho.

\subsection{Análisis de género y discurso histórico}

El análisis de género (Swales, 1990, 2004) ha demostrado ser un eficiente instrumento para el conocimiento y didáctica de la escritura científica. Estos estudios tienen como concepto base la "Movida Retórica" (Rethorical Move), definida como el extracto textual asociado a un propósito comunicativo que contribuye al logro del propósito global de un género (Swales, 1990, 2004). En este contexto, los estudios de Análisis de Género para los AI en historia son escasos. Entre estos destaca el trabajo de Holmes (1997) y Fuentes (2012). Holmes (1997) estudia contrastivamente la sección discusión en un corpus de AI de ciencia política, sociología e historia, en idioma inglés. Uno de los resultados relevantes de esta investigación es una MR exclusiva del discurso histórico, a saber: Establece consecuencias de los hechos más allá de las fechas estudiadas. En tanto, Fuentes (2012) estudia todas las secciones de un corpus de AI en historia y en español. En el estudio, se establece que las MR que predominan en el discurso en este corpus son: a) Narra un hecho, b) Interpreta un hecho, y c) utiliza una cita literal para referirse a otro autor o fuente. Además, el discurso histórico ha sido estudiado lingüísticamente en otro género, a saber, los manuales de historia (Oteíza, 2006, 2009a, 2009b). Estos trabajos analizan desde la Lingüística Sistémica Funcional (Halliday, 1984) los manuales en historia y específicamente el tratamiento del tema de la dictadura militar de Pinochet.

\subsection{Enfoque multidisciplinar a las conclusiones}

Desde distintas disciplinas se han propuesto consejos y restricciones, no sólo para la sección de conclusiones, sino para todas las secciones de un texto científico (Henríquez \& Zepeda, 2004; Valderrama, 2005, 2010; Cázares et al., 1999; Sánchez, 2011). Esta literatura funciona como manuales de escritura científica, por lo cual nos orienta hacia una percepción multidisciplinar de cómo se deben presentar retóricamente las 
conclusiones en un AI. Algunas restricciones que presenta esta literatura para las conclusiones, son: No se debe discutir, presentar los resultados ni concluir con datos no documentados en la investigación (Valderrama, 2005; Sánchez, 2011). Así también, se presentan sugerencias, tales como: se deben caracterizar los logros de la investigación, se deben exponer las ideas más relevantes de cada capítulo, debe haber un retorno a la introducción, se deben proyectar nuevas investigaciones, se debe evaluar la metodología y señalar los puntos fuertes y débiles de la investigación (Cázares et al., 1999; Valderrama, 2005; Sánchez, 2011). En términos narratológicos una conclusión o cierre es donde la historia llega a su fin (Propp, 1974). Desde la lingüística de género, y específicamente desde el trabajo de Swales $(1990,2004)$ se ha utilizado la teoría de MR para estudiar las conclusiones (Gnutzmann \& Oldenburg, 1991; Ciapuscio \& Otañi, 2002). Es así como el trabajo más completo desde el Análisis de Género con respecto a las conclusiones, y a pesar de su antigüedad, sigue siendo el trabajo de Gnutzmann y Oldenburg (1991). En este trabajo se analizan contrastivamente, desde el análisis de género, las conclusiones de dos revistas de lingüística en idioma inglés y otra en alemán. Otro trabajo desde el análisis de género analiza todas las secciones del AI en historia, realizando algunas menciones sobre las conclusiones en historia (Fuentes, 2012). Este trabajo, entre otras cosas, señala que no en todos los AI hay conclusiones. En estos casos el AI termina abruptamente con los resultados, lo que coincide con los resultados expuestos por Gnutzmann y Oldenburg (1991), y Ciapuscio y Otañi, (2002). También, desde esta misma teoría y con las MR para las conclusiones de Gnutzmann y Oldenburg, (1991) se han estudiado las conclusiones en un género similar, específicamente, el informe de investigación en estudiantes universitarios (Espejo, 2006). Además, se han estudiado las conclusiones desde la Lingüística Sistémica Funcional, para un corpus en español y en Paleontología (Ferrari, 2006). Entonces, y a partir de los estudios mencionados en esta sección, podemos señalar que las conclusiones, al igual que cualquier otra sección del AI manifestará variaciones por causas disciplinares, sociales, temporales y culturales.

\subsection{Modelo de MR en conclusiones en AI de historia}

A partir de lo expuesto en esta sección elaboramos un modelo de MR para describir las conclusiones de AI de historia. En este modelo incluimos las MR propuestas por Gnutzmann y Oldenburg (1991) para

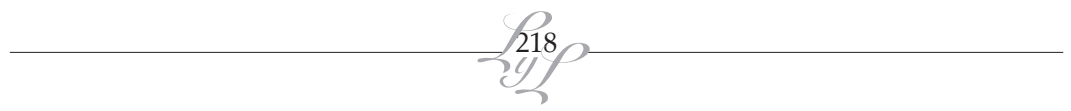


las conclusiones en lingüística, las MR propuestas para las conclusiones en el modelo de MR para AI de Sabaj, Toro y Fuentes (2011), las MR más frecuentes de los AI en historia de Fuentes (2012) y la MR de Holmes (1997) "Consecuencias futuras al hecho estudiado". A continuación, en la Tabla 1 presentamos el modelo de MR en la sección conclusiones del AI en historia:

1. Resumen de la investigación

2. Discute los resultados con otras investigaciones

3. Discusión de las fortalezas y debilidades de la investigación

4. Evaluación o relevancia de los resultados y sus implicancias

5. Interpreta resultados (hechos)

6. Narra un hecho

7. Cita literal a otra fuente

8. Consecuencias futuras al hecho estudiado

9. Señala si los resultados son coincidentes con la hipótesis

10. Ejemplifica los resultados de la investigación

Tabla 1: Modelo de MR para las conclusiones en AI de historia. Fuente: Gnutzmann y Oldenburg, 1991; Holmes, 1997; Sabaj, Toro y Fuentes, 2011; Fuentes, 2012

En la Tabla 1 apreciamos diez MR desde cuatro estudios de Análisis de Género.

\section{Materiales y métodos}

Nuestro corpus correspondió a las únicas tres revistas de historia indexadas en ISI, en Suramérica. Estas son la Revista Historia, de Chile; la Revista Historia Crítica, de Colombia y la Revista Quinto Sol, de Argentina. Con este criterio filtramos a las mejores revistas de historia según los evaluadores de ISI. Se determinó, como criterio, analizar los AI presentados para el año 2010 de estas tres revistas. A continuación, en 
la Tabla 2, se detallan las revistas, el país y la cantidad de AI en nuestro corpus:

\begin{tabular}{|l|c|c|}
\multicolumn{1}{|c|}{ Revista } & País & Cantidad \\
\hline Historia & Chile & 5 \\
\hline Historia Crítica & Colombia & 5 \\
\hline Quinto Sol & Argentina & 6 \\
\hline Total & & 16 \\
\hline
\end{tabular}

Tabla 2: Corpus de análisis

Los procedimientos realizados consistieron, en primer lugar, en la identificación de las conclusiones. En este sentido, y como señalamos anteriormente, las conclusiones no se presentan en todos los AI. Esto genera un problema para su identificación. En Análisis de Género este problema se identifica con las marcas límites (boundary markers) (Swales, 1990, 2004). Estas son las marcas textuales que identifican el fin o inicio de una MR o en este caso una sección. Para resolver este problema utilizamos el procedimiento de identificación de conclusiones implícitas declarado por Gnutzmann y Oldenburg (1991), utilizado también por Fuentes (2012), el cual consiste en identificar funciones comunicativas asociadas a las conclusiones en los últimos extractos o párrafos textuales de un AI. En este contexto, se identificaron conclusiones explícitas e implícitas, siguiendo los procedimientos de Fuentes (2012).

Luego de esta identificación, se procedió a etiquetar los textos por medio del software Atlas Ti. Las etiquetas o códigos se fundamentaron en un modelo de MR para conclusiones en historia creado para esta investigación (Tabla 1). De esta forma, abordamos elementos de las conclusiones, del género AI y del discurso científico de la historia. Una vez etiquetado el corpus se procedió a cuantificar los porcentajes de ocurrencia de todas las categorías en relación al total de AI analizados. Finalmente, se procedió a la interpretación de esta ocurrencia, su descripción lingüística, y ejemplificación de las MR más frecuentes.

\section{Resultados y discusión}

En esta sección presentamos los análisis de nuestro corpus, los cuales son los siguientes: Análisis de presencia de subtítulos y tipos de subtítulos para presentar las conclusiones; Análisis de frecuencia de las

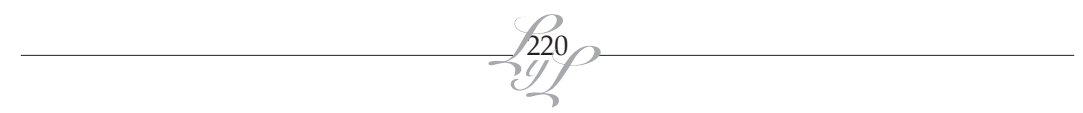


MR presentes en nuestro corpus y caracterización lingüística de las MR presentes.

\subsection{Análisis de presencia de subtítulos y tipos de subtítulos}

La presencia o ausencia de subtítulos para identificar el inicio de las conclusiones es un elemento de gran relevancia en la elaboración de un AI. Este hecho involucra lo que Swales $(1990,2004)$ ha denominado boundary markers (ver punto 3). La presencia de subtítulos identificatorios, los tipos de subtítulos, el total de conclusiones explícitas e implícitas y el total de conclusiones tanto presentes como ausentes para nuestro corpus se presentan en la Tabla 3:

\begin{tabular}{|c|c|c|c|c|}
\hline & \multicolumn{2}{|c|}{ Conclusiones Explícitas } & \multicolumn{2}{|c|}{ Conclusiones Implícitas } \\
\hline & $\begin{array}{c}\text { Subtítulo } \\
\text { Conclusiones } \\
\text { explícito }\end{array}$ & $\begin{array}{c}\text { Subtítulo } \\
\text { asociado a } \\
\text { conclusiones } \\
\text { explícito }\end{array}$ & Sin subtítulo & $\begin{array}{c}\text { Sin } \\
\text { conclusiones }\end{array}$ \\
\hline$\%$ & $43,75 \%$ & $31,25 \%$ & $18,75 \%$ & $6,25 \%$ \\
\hline $\begin{array}{c}\text { Total } \\
\text { explícitas e } \\
\text { implícitas } \\
\end{array}$ & \multicolumn{2}{|c|}{$75 \%$} & \multicolumn{2}{|c|}{$25 \%$} \\
\hline $\begin{array}{c}\text { Total } \\
\text { presentes } \\
\text { y ausentes }\end{array}$ & \multicolumn{2}{|c|}{$93,75 \%$} & \multicolumn{2}{|c|}{$6,25 \%$} \\
\hline
\end{tabular}

Tabla 3: Cantidad, porcentaje y tipo de subtítulos en las conclusiones en nuestro corpus

En la Tabla 3, observamos que el 75\% de nuestro corpus presenta las conclusiones explícitamente con el subtítulo "Conclusiones" o uno asociado a este, como por ejemplo: "Consideraciones finales", "Reflexiones finales", "A modo de cierre", "Palabras finales". En cambio, existió un $25 \%$ de AI sin un subtítulo claro que identificara el inicio de las conclusiones o no presenta conclusiones. Estos resultados son similares a los obtenidos por Fuentes (2012) en donde se identificaron un $80 \%$ de conclusiones explícitas y un $20 \%$ de conclusiones implícitas. De esta forma, encontramos que el 18,75\% de los AI que no presentan ningún tipo de subtítulo que identifique la presencia de conclusiones, pero sí 
en sus últimos tramos textuales se identifican funciones asociadas a conclusiones. En estos casos las conclusiones se sumaron al promedio de las conclusiones presentes. Además, con esta misma metodología identificamos un AI que no presenta ni subtítulo ni funciones asociadas a las conclusiones. Este AI continúa presentando resultados y termina en forma abrupta. Estos datos coinciden con los resultados de Gnutzmann y Oldenburg (1991) y Fuentes (2012). Entonces, si integramos 18,75\% de las conclusiones sin subtítulo al total de presencia de conclusiones tenemos que en nuestro corpus las conclusiones se presentan en un 93,75\%, lejos del 62,5\% de presencia obtenido por Fuentes (2012). Este hecho se puede explicar por la antigüedad del corpus de Fuentes (2012) en donde se recolectaron textos entre los años 2000 y 2008. Tal como se ha sugerido (Fuentes, 2012), la historia como disciplina científica se ha resistido a las imposiciones de formato que implica el discurso científico, específicamente, en el género AI. Contrastando los resultados de esta investigación, con aquellos de Fuentes (2012), podemos advertir que, en la actualidad, el discurso de la historia ha cedido a las imposiciones del discurso científico. Esta sería la razón por la cual, al analizar AI más actuales, podemos observar que la presencia de la sección de las conclusiones es mayor.

\subsection{Correspondencia del modelo con nuestros datos y presencia de las MR en la sección conclusión de AI en historia}

A continuación, en la Tabla 4, presentamos las MR presentes en las conclusiones de los AI de nuestro corpus. Así también, se presenta el grado de correspondencia del modelo de MR para el análisis de las conclusiones en historia con los datos obtenidos en la presente pesquisa:

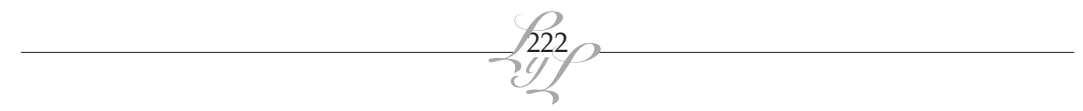




\begin{tabular}{|c|c|c|}
\hline $\begin{array}{c}\text { Presencia en } \\
\text { el Modelo }\end{array}$ & MR & $\begin{array}{c}\% \text { de AI con } \\
\text { esta función } \\
\text { en la sección } \\
\text { conclusiones }\end{array}$ \\
\hline Sí & Interpreta resultados (hechos) & $100 \%$ \\
\hline Sí & $\begin{array}{c}\text { Narra un hecho/ Resumen de la } \\
\text { investigación }\end{array}$ & $75 \%$ \\
\hline Sí & $\begin{array}{c}\text { Consecuencias futuras al hecho } \\
\text { estudiado }\end{array}$ & $37,5 \%$ \\
\hline Sí & $\begin{array}{c}\text { Discute sus resultados con otras } \\
\text { investigaciones }\end{array}$ & $31,25 \%$ \\
\hline Sí & Utiliza una cita literal de otra obra & $25 \%$ \\
\hline Sí & $\begin{array}{c}\text { Los resultados son concordantes con } \\
\text { las hipótesis }\end{array}$ & $18,75 \%$ \\
\hline Sí & $\begin{array}{c}\text { Ejemplifica los resultados de la } \\
\text { investigación }\end{array}$ & $12,5 \%$ \\
\hline Emergente & $\begin{array}{c}\text { Presenta las conclusiones de la } \\
\text { investigación }\end{array}$ & $12,5 \%$ \\
\hline Emergente & $\begin{array}{c}\text { Da cuenta de la importancia del tema } \\
\text { investigado }\end{array}$ & $12,5 \%$ \\
\hline Emergente & Necesidad de investigaciones futuras & $6,25 \%$ \\
\hline
\end{tabular}

Tabla 4: Correspondencia del Modelo con nuestros datos y porcentaje de AI que presentan estas funciones en la sección conclusiones

En la primera columna de la Tabla 4, podemos observar si las MR del modelo (Tabla 1) aparecen también en nuestro corpus de análisis. Tal como se observa, 8 de las $10 \mathrm{MR}$ del modelo aparecen también en nuestros datos. Este resultado sugiere queel modelo utilizado predice, en efecto, las características retóricas de la sección conclusiones en $\mathrm{AI}$ de historia. Así también, en el análisis emergieron 3 categorías no consideradas en el modelo, las que, sin embargo, aparecen solo en un $12,5 \%$ y en un 6,25\% de los AI analizados. Las dos MR con mayor presencia en nuestro corpus son "Interpreta resultados" y "Narra un hecho/ Resumen de la investigación" con un 100\% y un 75\% de presencia respectivamente. El resto de los propósitos comunicativos no supera el 37,5\% de aparición en los AI analizados. 


\subsection{Caracterización lingüística de las MR en las conclusiones de AI en historia}

En esta sección describiremos lingüísticamente una por una las MR presentes en nuestro corpus.

En la MR interpreta resultados (hechos), se pudieron identificar una serie de subtipos. Estos subtipos son funciones que adopta este propósito comunicativo. Los subtipos identificados se presentan en la Tabla 5:

\begin{tabular}{|l|c|}
\hline \multicolumn{1}{|c|}{ Interpreta resultados (hechos) } & $\%$ \\
\hline Consecuencia & $93,75 \%$ \\
\hline Categorización & $62,5 \%$ \\
\hline Juicios valóricos & $50 \%$ \\
\hline Causa & $31,25 \%$ \\
\hline
\end{tabular}

Tabla 5: Formas de interpretar resultados o hechos y porcentaje de aparición en la sección conclusiones de AI en historia

En la Tabla5, se muestran los porcentajes de aparición de estos subtipos respecto del total de los AI analizados. Así, podemos observar que en un 93,75\% de los AI analizados se interpretan los hechos recalcando en las consecuencias de los hechos. Este subtipo se manifiesta en formas como: "Entonces", "en definitiva”, "en consecuencia”, "asî". Los verbos están conjugados en pasado y suelen contener las explicaciones de un hecho anterior. En este sentido, este propósito necesita de un texto anterior que en esta ocasión va a ser explicado. Por su parte, la forma menos frecuente de interpretar hechos es a través de la textualización de la causas de un hecho. Esta categoría se identifica por la referencia directa a las causas u origen de un hecho, a través del uso de formas como "origen", "causa", "razón", entre otras. La interpretación de hechos como categorización se refiere a la delimitación de un ámbito o campo al cual se atribuye el hecho. Por ejemplo, se determina que un hecho se debe a factores sociales, culturales o laborales. Este subtipo se puede identificar a través de marcas de ordenamiento y/o enumeración (números o letras) y, por la presencia de algunos elementos paraverbales como el uso de comillas o negritas. En el 50\% de las conclusiones, la interpretación de hechos incluye la emisión de juicios valóricos. Este subtipo se identifica por textualizaciones, tales como: "es interesante", "es interesante constatar", "otra queja interesante", "doloroso" y "estremecedora". 
La MR "Narra un hecho" y "resumen de la investigación" se integraron en una sola, dado que se identifican con los mismos extractos textuales y, en este caso, cumplen la misma función, esto es, se narran hechos para resumir la investigación. Estos resultados se contraponen a lo señalado por Valderrama (2005) y Sánchez (2011) quienes señalan que en la conclusión no se deben resumir los resultados, o en otras palabras, la conclusión no es un resumen de la investigación. La característica lingüística principal de esta MR es el Modo de Organización Discursivo (en adelante MOD) Narrativo. El discurso se presenta como objetivo, sin poner en duda que el hecho sucedió de esa manera.

Con la eliminación de los corregidores y el reparto de mercancías en 1784, estos tejidos de relaciones comerciales comenzaron a funcionar plenamente en la región, impulsados por la expansión de los mayoristas peninsulares que se asociaban con intermediarios locales para despachar al Alto Perú y los mercados regionales los productos que controlaban. (CCAIH 3)

Como se aprecia en la cita, la utilización de verbos en pasado simple y formas impersonales como el infinitivo son características del discurso narrativo.

En las interpretaciones por "Consecuencias futuras al hecho estudiado" (Holmes, 1997) se puede observar que coincide con la idea de "Aceleración en la historia" (Barthes, 1994), en donde se evidencia la distancia temporal entre el narrador y el hecho narrado.

Sobre esta base se iba a poner en marcha, a partir de fines del siglo XIX, la vitivinicultura moderna en la región. (CCAIH 1)

En el ejemplo apreciamos que esta MR se identificó por la relación entre la fecha mencionada y la fecha de los objetivos. Es decir, involucra contenido y no forma para su identificación.

La MR “Discute sus resultados con otras investigaciones" coincide con la MR de Gnutzmann y Oldenburg (1991) "Resumen de investigaciones recientes en el área investigada". Esto se opone a lo establecido por Valderrama (2005) y Sánchez (2011) quienes señalan que en la conclusión no se discute. Junto con esto, en investigaciones sobre MR en AI (Sabaj, Toro \& Fuentes, 2011) se señaló que esta MR aparece tanto en la sección discusión como conclusiones. Esto cobra más importancia en nuestro 
corpus, debido a que en historia no se presenta la discusión como una sección, sino como MR, y por lo general, en las secciones de resultados y conclusiones (Fuentes, 2012).

Es así que en la construcción de la figura de Ceferino, aunque el resultado sea diverso de acuerdo a las distintas fuentes analizadas, hay una búsqueda de resolución de la tensión entre aboriginalidad y santidad por la vía de la transformación. (CCAIH 11)

En el ejemplo anterior observamos que no se realizan referencias directas a los trabajos con que se discute. De esta forma, se utilizan expresiones como "la literatura especializada" y "distintas fuentes analizadas". Además, la discusión suele superar o criticar más que apoyar las investigaciones anteriores. Este hecho se comprueba con textualizaciones tales como "Lejos del perfil descuidado e irracional que le atribuía la literatura especializada", "resulta insuficiente" y "aunque el resultado sea diverso de acuerdo a las distintas fuentes analizadas".

La MR “Utiliza una cita literal de otra obra” se presenta de dos formas, a saber: a) ejemplificando con una cita, y b) continuando el relato con una cita literal. Esto también ha sido constatado por Fuentes (2012).

Tal vez sea el epígrafe escrito en la tumba que alberga los restos del Capitán Andresen el que mejor sintetice la visión heroica que ha quedado en el imaginario colectivo de la región sobre los hombres de la Sociedad Ballenera de Magallanes: “Al Capitán Adolfo Andresen, 1863-1940 Que hizo flamear en la isla Decepción la bandera chilena en señal de soberanía afianzando los derechos de Chile a la Antártica, prolongación histórica y geográfica de la República. ¡Honor a su memoria! Punta Arenas, 1 Noviembre- 1949". (CCAIH 2)

Sin embargo, Gaspar era mestizo y en octubre de ese año decidía vender el solar que había heredado de su padre y que se encontraba "de la otra parte del Río desta ciudad". (CCAIH 5)

La característica común en estas dos formas de presentar una cita literal es la doble comilla en el extracto citado. Como se puede apreciar en los ejemplos, para la identificación de las formas planteadas para esta $\mathrm{MR}$, se necesita del extracto anterior de la cita. Ahora bien, en el primer ejemplo observamos cómo se utiliza una cita literal para ejemplificar un

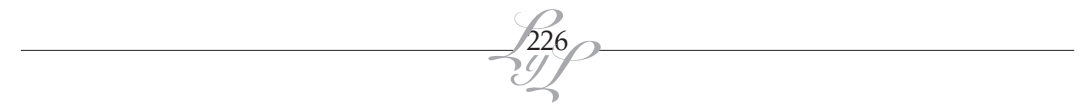


relato anterior. Esta función se identificó por medio de los dos puntos y por el extracto citado entre comillas. Además, el contenido nos entrega pistas para esta identificación, en tanto, el extracto citado es una síntesis de una idea que se intenta defender. En el segundo ejemplo se utiliza la cita literal para continuar un relato anterior. Esta función se identifica por la ausencia de conectores lingüísticos, presentación de la cita o dos puntos.

En la MR "Ejemplifica los resultados de la investigación" se logró identificar por la referencia explícita a la palabra ejemplo.

- por ejemplo, las modalidades históricas de su recepción agraria-. (CCAIH 10)

De esta forma, encontramos expresiones tales como: "otro ejemplo" y "por ejemplo".

La MR “Los resultados son concordantes con las hipótesis” involucra un metatexto, es decir, cuando un texto habla sobre el mismo texto. Si bien en esta MR se esperaría que se retomen otros elementos, principalmente desde las secciones iniciales del AI, tales como objetivos, metodologías, esto no sucede. Esto nos lleva a pensar que es más importante recordar la MR asociada a las hipótesis que la MR asociada a los objetivos y metodologías en las conclusiones en historia.

La evidencia documental ha permitido dar por superada las hipótesis anteriores. (CCAIH 1)

Los ejemplos muestran que la MR se caracteriza por llevar explícitamente la palabra hipótesis.

La MR "Presenta las conclusiones de la investigación" cumple la función de señalar explícitamente al lector el lugar del texto donde comienza esta sección. Este hecho podría tener extrema importancia en ocasiones en que no exista un subtítulo explícito que indique el inicio de las conclusiones como ocurre en el $25 \%$ de nuestro corpus.

De todo lo visto hasta ahora podemos concluir que el acceso de la mujer a la educación superior fue apoyado por las dos Españas (CCAIH 9).

Los ejemplos muestran que se hace una referencia explícita a las conclusiones, pero con distintos matices. Así, vemos expresiones como "Como se ha podido apreciar a lo largo de este trabajo", "De todo lo visto 
hasta ahora podemos concluir" y "... lleva a concluir". Estos resultados coinciden con Fuentes (2012).

En la MR "Da cuenta de la importancia del tema investigado" se justifica la importancia del tema investigado. Se valoran las fuentes, los resultados, los temas.

Este tipo de testimonio es diferente del construido para reivindicar una posición política en medio del conflicto, o para justificar acciones de guerra. El de Héctor es un testimonio de la violencia desnuda que es recordada en la vida de una persona como un proceso de violencia interminable. (CCAIH 8)

El ejemplo muestra que la importancia del tema investigado se realiza por medio de la distinción de originalidad de la fuente principal, a diferencia de otros trabajos. En el otro ejemplo se utiliza la expresión "brinda la posibilidad" para expresar la importancia del tema.

La MR "Aplicabilidad de los resultados" indica el aporte o implicancia de los resultados. Esta MR también fue identificada por Gnutzmann y Oldenburg (1991).

La reposición de estas tensiones es un aporte para la construcción de una imagen del pasado. (CCAIH 16)

En el ejemplo observamos que la identificación de esta MR se obtuvo por medio del verbo "aportar".

Finalmente, la MR "Necesidad de investigaciones futuras" se trata de las proyecciones científicas de la investigación.

Una última observación, que debería ser confirmada con una investigación sobre la recepción y uso de las imágenes de Ceferino: a pesar del rescate de la imagen fotográfica verificado en las publicaciones de las últimas décadas. (CCAIH 11)

El ejemplo da cuenta de que esta MR ocupa el verbo deber en condicional y el verbo confirmar.

\section{Conclusiones}

Con los resultados expuestos en la presente investigación observamos que la sección conclusiones no se manifiesta de la misma forma en las distintas disciplinas. Así, hemos podido constatar que las funciones

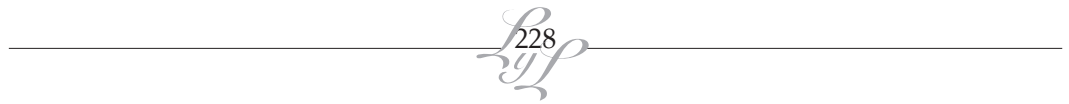


características de esta sección en la historia no necesariamente coinciden con lo que se espera en otras disciplinas (Valderrama, 2005; Sánchez, 2011). Así también, contrastando los resultados con aquellos obtenidos por Fuentes (2012), hemos evidenciado que a medida que pasa el tiempo, la historia ha comenzado a ceder a las presiones de formato requeridos comúnmente por el discurso científico. Esto se demuestra en el hecho que al analizar AI más actuales, aumenta la presencia de la sección conclusiones en historia.

Un tercer elemento de variación en las conclusiones es la variable cultural. Esta variable no fue parte de nuestros objetivos pero creemos que es una variable que influiría en la redacción de conclusiones en AI en historia. En otras palabras, creemos que existirían variaciones en la forma que se presentan las conclusiones de AI en historia en Latinoamérica a otras presentadas en Europa.

Esta investigación comprueba, una vez más, lo inseparable del discurso histórico del modo de organización del discurso narrativo. Además, hemos podido constatar que la narración se utiliza sobre todo para resumir la investigación. Aún así, este resumen de la investigación no se materializa en metatextos como volver a los objetivos o la metodología, sino más bien, en la narración en forma sintética de los principales hechos. En este contexto, creemos que las conclusiones son más que resumir los resultados. Principalmente, y suele haber consenso en la amplia literatura indagada en esta investigación, que las conclusiones debieran hablar más de las unidades fundamentales de la pesquisa: Los objetivos, las metodologías y las hipótesis, además de las implicancias empíricas y futuras de la investigación.

Metodológicamente, comprobamos que el modelo construido a priori para describir la sección de las conclusiones en AI de historia predice de forma consistente las funciones que en efecto se encuentran en esta sección. Recordemos que este modelo se realizó con los aportes desde el análisis de género, estudios específicos para las conclusiones y criterios de la epistemología de la historia. En este sentido, creemos que la variable epistemológica es necesaria en futuros estudios sobre MR. Los resultados de esta investigación son útiles para quienes se interesen en los estudios sobre la sección conclusiones y en el discurso científico de la historia. Además, son de gran ayuda para la didáctica de la escritura científica. La gran diversidad de formas de interpretación develadas en la presente investigación abre las puertas a futuras profundizaciones sobre el tema. 


\section{Bibliografía}

Barthes, R. (1994). El susurro del lenguaje. Barcelona: Paidós.

Cázares, L.; Christen, M.; Jaramillo, E.; Villaseñor, L. \& Zamudio, L. (1999). Técnicas actuales de investigación documental. México: Trillas.

Ciapuscio, G. \& Otañi, I. (2002). “Las conclusiones de los artículos de investigación desde una perspectiva contrastiva". RILL 15, 117-133.

Charaudeau, P. \& Maingueneau, D. (2005). Diccionario de Análisis del discurso. Buenos Aires: Amorrortu.

Chartier, R. (2005). El mundo como representación. Barcelona: Gedisa.

Danto, A. (1965). Analytical Philosophy of History. Cambridge: Cambridge University Press.

Espejo, C. (2006). “La movida concluyendo en torno al tema en informes de investigación elaborados por estudiantes universitarios". Onomázein 13 (1), 35-54.

Ferrari, L. (2009). “Marcadores de modalidad epistémica y evidencial en el análisis de las conclusiones de artículos de investigación de distintas disciplinas". Revista ALED 9 (2) 5- 25.

Fuentes, M. (2012). “El discurso científico de la historia: Análisis estructural y retórico de los artículos de investigación en historia". Boletín de Filología. 47 (1). [En prensa]

Gnutzmann, C. \& Oldenburg, H. (1991). “Contrastive Text Linguistics in LSP-Research: Theoretical Considerations and some Preliminary Findings". En Schröder, H. Subjectoriented Texts: Language for Special Purposes and Text Theory. New York/Berlin, Walter de Gruyter: 101-136.

Henríquez, E. \& Zepeda, M. (2004). Elaboración de un artículo científico de investigación. Ciencia y Enfermería 10 (1), 17- 21.

Holmes, R. (1997). "Genre Analysis, and the Social Sciences: An Investigation of the Structure of Research Article

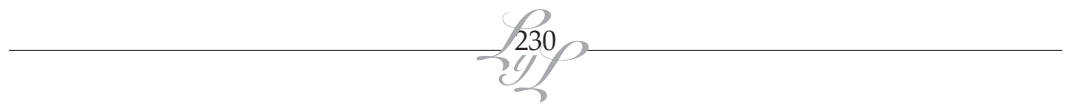


Discussion Sections in Three Disciplines". English for Specific Purposes 16 (4), 321-337.

Hyland, K. (2005). Metadiscourse. London: Continuum.

Koselleck, R. (2010). historia/ Historia. Madrid: Trota.

Lozano, J. (1994). El discurso histórico. Madrid: Alianza.

Propp, V. (1974). Morfología del cuento. Caracas: Fundamentos.

Swales, J. (1990). Genre Analysis. English in academic and research setting. Cambridge: Cambridge University Press.

Sánchez, A. (2011). Manual de redacción académica e investigativa: Cómo escribir, evaluar y publicar artículos. Medellín: Católica del norte Fundación Universitaria.

Swales, J. (2004). Research Genres: Exploration and Aplications. Cambridge: Cambridge University Press.

Ordóñez, L. (2008). “Historia, literatura y narración”. Bogotá, Revista Historia Crítica 36, 194- 222.

Ortega y Gasset, J. (1983). Obras completas. Madrid: Alianza.

Oteíza, T. (2006). El discurso pedagógico de la historia. Un análisis lingüístico sobre la construcción ideológica de la historia de Chile (1970-2001). Santiago de Chile: Frasis.

. (2009a). "Solidaridad ideológica en el discurso de la historia: Tensión entre orientaciones monoglósicas y heteroglósicas". Revista Signos 42 (70), 219-244.

. (2009b). “Cómo es presentada la historia contemporánea en los libros de textos chilenos para la escuela media". Revista Discurso y Sociedad 3 (1), 150-174.

Sabaj, O.; Toro, P. \& Fuentes, M. (2011). “Un modelo de Movidas Retóricas para el análisis de artículos de investigación en español". Revista Onomázein 24 (2), 245-271.

Valderrama, J. (2010). "Preparación de un artículo para ser publicado en la revista internacional formación universitaria". Información Tecnológica 3 (1), 31-38. 
. (2005). "Principales aspectos sobre la preparación de un artículo para ser publicado en una revista internacional de corriente principal". Información Tecnológica 16 (2), 3- 14.

Veyne, P. (1984) Cómo se escribe la historia. Madrid: Alianza.

White, H. (2003). El texto histórico como artefacto literario. Barcelona: Paidós.

. (2010). Ficción histórica, historia ficcional y realidad histórica. Buenos Aires: Prometeo. 